\title{
Intensive physiotherapeutic management of congenital muscular torticollis
}

\author{
S S Wakista ${ }^{1}$, G Ranchagoda ${ }^{2}$ \\ Sri Lanka Journal of Child Health, 2004; 33: 43-5
}

(Key words: Torticollis, sternocleidomastoid tumour, physiotherapy)

\begin{abstract}
Objectives To assess effectiveness of early and intensive physiotherapeutic management of congenital muscular torticollis and determine number of visits to physiotherapy department to achieve maximum clinical improvement in relation to age of initiation of treatment.
\end{abstract}

Setting Physiotherapy department, Base Hospital, Horana.

Method A prospective study was done from May to December 2002 on babies brought for treatment of congenital muscular torticollis. Intensive physiotherapy was given by a physiotherapist twice daily for 1 week and the mothers instructed in the procedure. Further physiotherapy was given by mothers at home with regular visits to the physiotherapy department. On complete resolution of torticollis, babies were referred back to the well baby clinic where they were followed up until one year of age. For statistical evaluation, Student's t-test was used.

Results 19 babies, ages ranging from 18-90 days, had been referred for treatment of congenital muscular torticollis during study period. 11 were female. 16 were diagnosed during first 2-3 weeks of life, and 3 at age of 3 months. 11 had left sided sternocleidomastoid tumour, 7 had right sided tumour and one had sternocleidomastoid shortening without a palpable tumour. There were 17 spontaneous vaginal deliveries (16 cephalic, 1 breech) and 2 vacuum extractions.

In 14 cases mothers were primigravidae. Infants in whom treatment was initiated within 4 weeks of birth achieved maximum clinical improvement with a mean of 12 (SD 3.3) visits to physiotherapy department. Infants treated after 4 weeks achieved

${ }^{1}$ Lecturer, Disability studies unit, Faculty of Medicine, University of Kelaniya, ${ }^{2}$ Consultant Paediatrician, Base Hospital, Horana.

(Received on 15 November 2003) maximum clinical improvement with a mean of 22 (SD 6.4) visits to physiotherapy department. This is statistically significant $(\mathrm{t}=3.81 . \mathrm{p}<0.01)$

Conclusions Congenital muscular torticollis is correctable if detected early and physiotherapy commenced forthwith. Awareness programmes for parents and their active participation are essential in terms of full recovery and reduction of number of visits to physiotherapist.

\section{Introduction}

Torticollis or "wry neck" is a condition in which the infant or child holds the head in a rotated, tilted position ${ }^{1}$. When torticollis is recognised in the first 6 8 weeks of life, the usual cause is congenital muscular torticollis ${ }^{2}$. If the infant is examined within the first month of life, a mass or "tumour" is usually palpable in the neck ${ }^{3}$. It is generally a non-tender, soft enlargement that is mobile beneath the skin and attached to, or located within, the body of the sternocleidonastoid muscle. The mass attains maximal size within the first month and then gradually regresses ${ }^{2}$. If the child is examined at 4-6 months of age, the tumour is usually absent and the contracture of the sterno-cleidomastoid muscle and torticollis are the only clinical findings ${ }^{2}$.

\section{Objectives}

To assess effectiveness of early and intensive physiotherapeutic management of congenital muscular torticollis and

determine number of visits to physiotherapy department to achieve maximum clinical improvement in relation to age of initiation of treatment.

\section{Method}

A prospective study was done from May to January 2002 on babies brought to physiotherapy department, Base Hospital, Horana for treatment of congenital muscular torticollis. The following physiotherapeutic procedure was carried out on these babies: 
1. Application of baby ointment on neck.

2. Gentle massage of tumour with thumb (thumb kneading). At same time parents were instructed in this procedure.

3. Rotation of neck to affected side with gentle traction by a physiotherapist.

4. Keeping infant in prone position during rest with neck rotated to side of lesion. To keep neck in this position, sand bags or pillows were used as supports.

5. Mother was instructed to keep ear of nonaffected side of baby away from breast when feeding (neck must be rotated towards affected side).

6. To induce active movements to side of lesion external stimuli such as sounds and toys were used.

In first week, exercises were done twice a day by a physiotherapist and parents instructed in the procedure. Once the physiotherapist was satisfied that the parents were able to work with their children, exercises were permitted to be carried out at home. Initially they were asked to visit physiotherapist twice a week. On positive clinical improvement visits were gradually reduced to once a week, once a fortnight and once a month. Reassessment was done monthly up to 6 months. All patients were given diagnosis cards and directed to well baby clinic for further assessment and follow up till one year of age.

For statistical evaluation, Student's unpaired t-test was used.

\section{Results}

19 babies, ages ranging from 18-90 days, had been referred to the physiotherapy department, Base Hospital, Horana for treatment of congenital muscular torticollis during study period. 11 were female and 8 were male. 16 were diagnosed during the first 2-3 weeks of life, and 3 at the age of three months. 11 had left sided sternocleidomastoid tumour, 7 had right sided tumour and one had sternocleidomastoid shortening without a palpable tumour. There were 17 spontaneous vaginal deliveries (16 cephalic, 1 breech) and 2 vacuum extractions. There were 8 difficult deliveries due to obstructed labour. In 14 cases mothers were primigravidae.
No sternomastoid tumour or torticollis was identified in any of the 19 babies by 6 months of age.

14 babies, assessed one year from the termination of treatment, did not show any recurrence of torticollis. In 7 infants, where treatment was initiated within 4 weeks of birth, maximum clinical improvement was achieved with a mean of 12 (SD 3.3) visits to physiotherapy department. 12 infants, treated after 4 weeks, achieved maximum clinical improvement with a mean of 22 (SD 6.4) visits to physiotherapy department. This is statistically significant $(\mathrm{t}=3.81$. $\mathrm{p}<0.01$ )

\section{Discussion}

Congenital muscular torticollis is not accompanied by bone abnormalities or neurologic deficit and is believed to be caused by ischaemia of the sternocleidomastoid muscle, particularly the sternal head, because of intrauterine positioning or increased pressure during passage through the birth canal ${ }^{3,4,5}$. A recent hypothesis suggests that the disorder could be the result of a perinatal compartment syndrome of the sternocleidomastoid muscle ${ }^{6}$.

If detected early, more than $90 \%$ of congenital muscular torticollis can be managed with a stretching programme performed by parents after instruction by physiotherapist ${ }^{1,3,4}$. The head is gently manipulated in a manner that stretches the contracted muscle. Objects and toys can be positioned to encourage neck motion away from the contracture ${ }^{1,2}$. Provided that stretching is started early in life, most cases can be treated conservatively ${ }^{7}$. In children not receiving early treatment, deformity of the skull and facial asymmetry gradually develop, called plagiocephaly ${ }^{1}$. The few infants who fail to improve with stretching by 1 year of age require surgical release of the sternocleidomastoid muscle1. Surgery consists of open division of the sternocleidomastoid muscle and corrects the head position and improves plagiocephaly in more than $50 \%$ of patients. Surgical outcomes are best when performed in children 3-5 years of age ${ }^{8}$.

An important aspect of management is reassuring parents. In all cases anxiety and nervousness of parents had been observed. Many parents believed the treatment procedure may cause pain and harm to their children. Certainly in many cases the parents needed professional counselling. Active participation and regular attendance of the parents in physiotherapeutic management is essential and had been achieved by educating them to perform safe exercises and neck positioning. 
Commencement of early treatment is essential. There was a statistically significant difference regarding visits to physiotherapy department between children treated within 4 weeks of birth and those treated later.

\section{Conclusions}

1. Congenital muscular torticollis is correctable if detected early and physiotherapy commenced forthwith.

2. Awareness programmes for parents and their active participation are essential in terms of full recovery and reduction of number of visits to physiotherapist.

\section{Acknowledgements}

We thank Mrs Janaki Abeyratne, residential physiotherapist, Base Hospital, Horana for her participation in physical management and parent education.

\section{References}

1. Epps H R, Salter R B. Orthopaedic conditions of the cervical spine and shoulder. Paediatric Clinics of North America 1996; 43: 919-31.
2. MacDonald D. Sternomastoid tumour and muscular torticollis. J Bone Joint Surg Br 1969; 51: 432-43.

3. Cheng J C Y, Au A W Y. Infantile torticollis: A review of 624 cases. J Pediatr Orthop 1994; 14: 802.

4. Hensinger R N. Orthopaedic problems of the shoulder and neck. Paediatric Clinics of North America 1986; 33: 1495-509.

5. Ling C M, Low Y S. Sternomastoid tumour and muscular torticollis. Clin Orthop 1972; 86: 14450.

6. Davids J R, Wenger D R, Mubarak S J. Congenital muscular torticollis: Sequela of intrauterine or perinatal compartment syndrome. J Pediatr Orthop 1993;13: 141.

7. Binder H, Eng G D, Gaiser J F et al. Congenital muscular torticollis: results of conservative management with long-term follow up in 85 cases. Arch Phys Med Rehabil 1987; 68: 222-5.

8. Wirth C J, Hagena F W, Wuelker N. Biterminal tenotomy for the treatment of congenital muscular torticollis. J Bone Joint Surg Am 1992; 74: 427. 
